\title{
GCU
}

Glasgow Caledonian

University

University for the Common Good

\section{Advances in image acquisition and processing technologies transforming animal ecological studies}

Nazir, Sajid; Kaleem, Muhammad

Published in:

Ecological Informatics

DOI:

10.1016/j.ecoinf.2021.101212

Publication date:

2021

Document Version

Author accepted manuscript

Link to publication in ResearchOnline

Citation for published version (Harvard):

Nazir, S \& Kaleem, M 2021, 'Advances in image acquisition and processing technologies transforming animal ecological studies', Ecological Informatics, vol. 61, 101212. https://doi.org/10.1016/j.ecoinf.2021.101212

\section{General rights}

Copyright and moral rights for the publications made accessible in the public portal are retained by the authors and/or other copyright owners and it is a condition of accessing publications that users recognise and abide by the legal requirements associated with these rights.

Take down policy

If you believe that this document breaches copyright please view our takedown policy at https://edshare.gcu.ac.uk/id/eprint/5179 for details of how to contact us. 


\title{
1 Advances in Image Acquisition and Processing 2 Technologies Transforming Animal Ecological 3 Studies
}

\author{
Sajid Nazir ${ }^{1}$, Muhammad Kaleem² \\ ${ }^{1}$ School of Computing, Engineering and Built Environment, Glasgow Caledonian University, Glasgow, UK \\ ${ }^{2}$ Department of Electrical and Computer Engineering, COMSATS University, Islamabad, Pakistan \\ Corresponding author: Sajid Nazir (sajid.nazir@gcu.ac.uk).
}

Abstract: Images and videos have become pervasive in ecological research and the ease of acquiring image data and its subsequent processing can provide answers in research areas such as species recognition, animal behavior, and population studies which are critical for animal conservation and biodiversity. Technological advances in imaging are enabling data collection from new areas such as from underwater, new modalities such as thermal and new ways of processing such as deep learning. These advances are accelerating due to ease of data collection, better storage and processing technologies with associated lowering costs. The advancements in state-of-the-art machine learning for image and video classification and analysis can directly be applied in ecology. Ecological applications are generally conducted in remote and harsh deployment environments, and therefore present formidable challenges that require appreciation of the limitations of such technologies. The ecological field is poised to make use of images acquired through drones, robotics, and satellites through machine learning for rapid advancements in critical research areas. Timely insights from such data help to understand and protect the species and environment. This paper provides a review of the advancements in image acquisition and processing technologies used in animal ecological studies. We also discuss concepts and technologies that would help foster future ecological research methodologies potentially opening new insights and quickening growth to an already rich and dataintensive field.

Keywords animal behaviour, big data, computer vision, deep learning, image processing, population monitoring, drone, robot

\section{INTRODUCTION}

The use of digital technologies has seen an uptake in virtually all ecological domains, enabling significant efficiencies in time, effort and cost. This has specifically transformed not only the way that the images and videos are captured in the field but more importantly how this complex data can be processed to infer statistics answering the research question.

Ecological research depends on data collection under difficult circumstances in order to extract meaningful inferences. Not taking the factors into consideration can result in systems providing a degraded performance or erroneous data. Ecological research often occurs in harsh and hostile environments in far-flung areas and may continue over many years (Nazir et al., 2016). Without large scale adoption of the digital tools and informatics makes the ecological research quite hard, costly and time-consuming if not downright impossible. The events of interest in ecological studies are often difficult to observe (Huang et al., 2014). The event to be recorded might only occur once during a whole season or year, or could occur sporadically. The data from the field can be collected through use of sensors which could provide a simple scalar value (number), e.g. GPS coordinate, or it could be a camera (image sensor) for capturing image or video.

We use the term image in this text to encompass all the image modalities such as infrared, thermal and also video. We consider image processing in this text to include the low level processing of images such as background subtraction, traditional machine learning such as Support Vector machines (SVM), and the current deep learning algorithms. Image processing on field images have challenges. Illumination and background changes make animal detection difficult (Weinstein, 2017). Data cleaning is required as challenging terrains and conditions make sensors fail (Dietterich, 2009). Ecological data often comprises of noisy data (and small datasets), which could be challenging (Shan et al., 2006). The sensors 
and deployments are sometimes inaccessible and often are in harsh weather conditions, with cold, humidity, and rain etc., taking its toll on the device's electronics. The monitoring may cover a large area (Rovero et al., 2013; Tabak et al., 2019). Errors and biases can be affected by environmental conditions or camera features (Beauxis-Aussalet and Hardman, 2015).

Camera trap images are affected by weather conditions, shadows and lighting conditions (Norouzzadeh et al., 2018). Natural environments pose many challenges such as tree movements by wind, camera movements (Dickinson et al., 2008; Newey et al., 2015) which can result in false positives. False positive (FP) images do not have an animal in them, creating a lot of extra work and problems as these have to be separated from other images of interest with an animal (Swinnen et al., 2014). Camera trap animal images pose challenges for image analysis due to background clutter; occlusion, poor illumination and animal pose (Yu et al., 2013). A study in Mohave Desert had complex backgrounds, plants swaying in breeze, and occlusions from vegetation to increase the challenges posed to computer vision techniques (Wilber et al., 2013). However automated image processing techniques can help overcome the difficulties in analysing the large datasets.

Ecology is being revolutionised through the use of digital technologies over last decades (Grémillet et al., 2012). Ecological research is data intensive in both time and space, with studies carried over large geographical areas and durations, while further exacerbated by volumes of data collection possible through ease, frequency and scale of data generation tools. Large scale data can now be gathered through much superior digital data acquisition tools which if unmatched by faster automated analysis to extract meaningful insights and information, would become obsolete and irrelevant. The automated digital data collection using camera traps and drones can generate an enormous amount of data that requires automated processing to extract the requisite information. Animal-borne imaging can provide ecological information collection that other technologies do not offer (O’Connell et al., 2020).

The focus of this paper is to provide a review of image acquisition and processing technologies in animal ecology studies. We also provide future directions, opportunities and challenges for the ecological research.

Rest of the paper is structured as follows: The preliminary information on image modalities and processing techniques are provided in Section 2. The image acquisition technologies are covered in Section 3 that also includes studies utilising basic image processing. Section 4 covers the studies reporting advanced image processing and analysis. Future research directions and challenges are covered in Section 5. Finally conclusion is provided in Section 6.

\section{Background}

\subsection{Image and Video Data}

An image is a two dimensional representation of intensity measure corresponding to each point in a scene. The number of pixels in the image is referred to as image resolution and is expressed as width $x$ height of the image. Images have a very important role in the research studies and provide a wealth of information with video also becoming common. The video could be considered as a succession of images such as Motion JPEG but commonly advanced video compression formats such as H.264 are used. Depending on the video resolution and frames per second a lot of data gets generated and requires resources for storage, processing and analysis. Diverse information can be gleaned from high resolution video with a wider field of view (Mattern et al., 2018). For example, high resolution satellite images of the earth make it possible to identify small objects (McMahon et al., 2014). The images of interest in ecology can be captured using any image modality: visible, infrared, radar (Stepanian et al., 2014; Schmaljohann, 2020) or thermal (Dahlen and Traeholt, 2017).

Visible light or infrared cameras although are passive devices but require an illumination source. Infrared cameras are normally used for capturing images at night, and infrared images are sometimes erroneously referred to as a grey scale images. A grey scale image has only a single channel (8 bits) to represent each pixel whereas IR image has 3 (RGB, 24 bits) channels. Optical cameras have an IR filter (Anderson et al., 2019) which can be removed to allow night time imaging with IR light, however the removal impacts the quality of daytime images, giving a washed-out appearance. Commercial camera traps have a movable infrared filter that moves out of line from the camera lens on sensing darkness. Thermal cameras on the other hand are passive devices and do not require an illuminating source and can capture the heat intensity radiated from the objects in the scene. Thermal cameras can provide better animal detection based on temperature differential between the animal and the ambient temperature. Radar images are dependent on active transmission of radar signal and its reception after reflection from a target. 
The capture of image and video requires a large amount of storage, processing, and battery resources at the capture platform such as camera traps, drones, and animal-borne sensors. Thus in order for a judicious use of resources, image capture can be started on determining the onset of a condition, such as presence of an animal in the field of view, or another trigger such as passage of a fixed amount of time as for time lapse images. The image capture devices can generate a large amount of data very quickly that needs automated processing to extract information at low cost and in less time.

\subsection{Basic Image Processing Techniques}

Some form of image processing is invariably required in all cases of image analysis. This could be the change of image resolution to suit a particular technique. Such pre-processing operations help to improve image feature quality, exclude empty images, or for colour balancing/enhancement.

Ecological studies are aided by an automatic identification of an animal in an image, video, which is the first step for other research objectives such as population estimation. Classification is identifying and segregating images based on a similarity criteria or features, and could be used to segregate false positive (FP) images, species, day and night photos etc. Object detection in dynamic and highly cluttered backgrounds is a difficult problem (Weinstein, 2017). This could be accomplished with background subtraction with thresholds, and colour matching (Koniar et al., 2016). The study by Swinnen et al. (2014) was aimed to filter out images and videos that were either empty or contained non-target species, thus reducing the amount of time required for manual processing. MATLAB was used to process the images and videos, and based on pixel changes from frame to frame and the animal size, motion was inferred. The threshold was set such that it should not eliminate any true target and might not discern all false positives but still considered useful to reduce manual processing.

The presence of an animal can be inferred by image differencing or background subtraction techniques. Image processing was used to segregate FP images in a camera trap study (Nazir et al. 2017). The benefit of basic techniques is that they are of low complexity and can be easily understood and implemented. In the simpler of case, two corresponding images can be used for an image difference to determine the foreground object whereas for more complicated cases a background model needs to be maintained.

\subsection{Advanced Techniques}

Computer vision is aimed at extracting high level information from images, such as to determine if an animal is present in an image. Computer vision nowadays heavily draws on machine and deep learning owing to their demonstrated successes (Krizhevsky et al., 2017). A survey of Computer Vision in ecology studies, were categorised into description, counting and identification (Weinstein, 2017).

Machine learning is a field of artificial intelligence, and provides better results compared to the conventional computer vision low level processing techniques. Deep learning is a sub-field of machine learning that has recently been popularised by impressive results for object recognition in images (Krizhevsky et al., 2017). Deep learning techniques are based on Artificial Neural Networks (ANNs) that mimic the human neural system and the depth of the network refers to the many hidden layers of processing. A review of deep learning applications in the field of ecology highlights the benefits to ecological disciplines surmising that deep learning will become a necessity in view of the large scale datasets being generated. Some application areas and recommendations for ecologists are also provided by Christin et al., (2019). A review of deep learning methods useful for species identification is provided in (Waldchen and Mader, 2018). However, deep learning techniques differ significantly from the more traditional approaches in machine learning.

The major difference between traditional machine learning versus deep learning is that traditional machine learning feature extraction is under human supervision whereas deep learning can extract features automatically (Christin et al., 2019). The most relevant deep neural network for image classification is Convolutional Neural Network (CNN) (Krizhevsky et al., 2017) which requires optimisations of hyper parameters and use of speedup techniques (Nazir et al., 2020). The earlier layers in a CNN learns low level features such as edges and lines whereas later layers learn higher level features. The training of the model requires large amount of processing time and resources and is often done using a Graphical Processing Unit (GPU) to reduce the time. After a model has been trained it can be tested quickly to classify an animal. Although a customised model can be designed and trained but it is possible to reuse a pre-trained model designed for another classification problem and re-use it for a new problem. 
One avenue in deep learning is investigating application of transfer learning from one image dataset to another (Norouzzadeh et al., 2018). Transfer learning made accessible by Google Cloud Machine Learning platform will pave the way of its usage (Weinstein, 2017). Transfer learning means that we reuse the pre-trained weights from a trained model whereas the original model may have been for a particular image resolution and a particular dataset but by changing the layers in the network we can adapt it to work on a new problem.

\section{IMAGE ACQUISITION}

Animal behaviour can be affected by human presence or even its perception by the animal under study. Remote capture of images and videos thus has a special significance in ecology. In this Section, we cover the four data acquisition technologies commonly used for image acquisition that is, camera traps, video loggers, drones and satellites, and their application in studies utilising basic image processing. A summary of the image acquisition techniques is provided in Table 1.

\subsection{Camera Traps}

Camera trap is a device that acquires data on sensing a physical phenomenon, for example, taking a picture on sensing motion, or vibration. The recording of an event could also be time triggered, that is time lapse or may be continuous. Commonly used sensor for detecting a motion event is the Passive Infrared (PIR) sensor that operates by sensing the movement of infrared (heat) energy radiated from warm bodies. Other sensors sometimes used for presence sensing are radar (Glen et al., 2013; Nazir et al., 2017) or active infrared (Caravaggi et al., 2017). However, there are other sensors such as vibration that can be used. A review of camera trapping for conservation behaviour research is provided in (Caravaggi et al., 2017). A review of camera trap use for wildlife studies describes technical aspects of the commercially available camera traps and usage of these devices in ecological studies (Burton et al., 2015). The accuracy in sensing can help reduce or eliminate false positives that are images without an animal in them. The false positives were reduced by using radar sensing and image processing on the camera trap unit (Nazir et al., 2017).

Camera trap is a non-invasive tool suitable for use in all types of terrain and weather conditions and can be used successfully to document difficult events (Huang et al., 2014). Camera traps have progressed from the earliest trap reported in 1890 that used mechanical activations, using trip wires, to the latest models with sophisticated electronics and processing (Kucera and Barrett, 2011). The camera traps have been extensively used in ecological field studies. These could be used for activity patterns, occupancy modelling and abundance estimation (O’Brien, 2011).

Camera traps were used to capture animal behaviour in response to anthropogenic disturbances by integrating it with a motion activated speaker (Suraci et al., 2016). Variations in pixel values and environmental conditions were used to identify empty recordings and other species in camera trap videos where the species of interest was Eurasian beaver (Castor fiber) (Swinnen et al., 2014).

Cliff nesting seabirds' population was monitored with computer vision, using time lapse images (Dickinson et al., 2008). Monitoring through manual counting would be laborious, and impractical. The authors employed background subtraction by using Mixture of Gaussian (MoG) for background modelling. However the proposed method was computationally intensive making real-time processing even at low resolution difficult and model initialisation was also difficult (Dickinson et al., 2008). Camera traps data is often combined with other data. Distribution, occupancy, and habitat associations of sengi (Rhynchocyon udzungwensis) were studied using camera traps in (Rovero et al., 2013) and analysed together with satellite data.

The commercial camera traps have been successfully used in ecological studies. There could be problems in ecological studies due to the limitations of low-end commercial recreational camera traps in deployment, operational and data management (Newey, 2015). Considering such constraints there have been some efforts to develop customised camera traps (Glen, 2013; Nazir et al., 2017).

A study describing investigation of a low-cost camera trap specification for small mammals covered different trigger speeds utilizing microwave sensors (Glen, 2013). It also describes efforts to standardise the field of view of the cameras by actively triggering the PIR and studying the resulting image. The study compared a commercial camera with three variants of a custombuilt model (Glen, 2013). An open access camera trap was developed that allowed for the operational features to be 


\begin{tabular}{|c|c|c|c|}
\hline $\begin{array}{l}\text { Image } \\
\text { Acquisition } \\
\text { Technology }\end{array}$ & Description/Purpose of Study & Imaging Device & Reference \\
\hline \multirow{3}{*}{$\begin{array}{l}\text { Camera } \\
\text { Trap }\end{array}$} & $\begin{array}{l}\text { Manual identification of scavengers preying on the carcass of a Golden Snub- } \\
\text { Nosed Monkey (Rhinopithecus roxellana) }\end{array}$ & Reconyx PC900 & Huang et al., 2014 \\
\hline & $\begin{array}{l}\text { Abundance monitoring of jaguar population over } 14 \text { years with manual identification } \\
\text { of individuals using open population models }\end{array}$ & $\begin{array}{l}\text { CamTrakker, Cuddeback } \\
\text { and DeerCam }\end{array}$ & $\begin{array}{l}\text { Harmsen et al., } \\
2017\end{array}$ \\
\hline & $\begin{array}{l}\text { Time saving in processing camera trap videos of Eurasian beavers (Castor fiber) to } \\
\text { identify and segregate empty recordings or other species; using Pixel value variation } \\
\text { and environmental conditions }\end{array}$ & Bushnell Trophy cams & $\begin{array}{l}\text { Swinnen et al., } \\
2014\end{array}$ \\
\hline $\begin{array}{l}\text { Custom } \\
\text { camera trap }\end{array}$ & $\begin{array}{l}\text { Scavenger behaviour with motion detection and image differencing at deer carcass } \\
\text { with images automatically back loaded through on-site satellite link }\end{array}$ & $\begin{array}{l}\text { Raspberry Pi Camera, } \\
\text { Bushnell }\end{array}$ & Nazir et al., 2016 \\
\hline \multirow{3}{*}{$\begin{array}{l}\text { Biologging } \\
\text { Sensor }\end{array}$} & Scavenging behaviour of Tasmanian devil using video collar & $\begin{array}{l}\text { GoPro Hero } 3 \text { White video } \\
\text { cameras }\end{array}$ & $\begin{array}{l}\text { Anderson et al., } \\
2019\end{array}$ \\
\hline & Foraging behaviour of yellow-eyed penguin with high definition video logger & $\begin{array}{l}\text { HD video logger, time-depth } \\
\text { recorder and GPS logger }\end{array}$ & $\begin{array}{l}\text { Mattern et al., } \\
2018\end{array}$ \\
\hline & Behaviour ecology of sharks with a custom animal-borne imaging system & $\begin{array}{l}\text { 720p Water } \\
\text { Wolf underwater camera, } \\
\text { 1080p Go Pro Hero } 4 \\
\text { camera }\end{array}$ & $\begin{array}{l}\text { O’Connell et al., } \\
2020\end{array}$ \\
\hline \multirow{4}{*}{ Drone } & Estuarine Crocodile (Crocodylus porosus) nesting sites localisation & Model S100, Canon & Evans et al., 2016 \\
\hline & $\begin{array}{l}\text { Behaviour responses of mallards Anas platyrhynchos, wild flamingos } \\
\text { (Phoenicopterus roseus) greenshanks (Tringa nebularia) }\end{array}$ & Phantom drone & Vas et al., 2014 \\
\hline & Detection of wild orangutans using thermal camera & $\begin{array}{l}\text { DJI Phantom } \\
\text { 4Pro, DJI Inspire } 1 \text { V2.0 }\end{array}$ & $\begin{array}{l}\text { Dahlen and } \\
\text { Traeholt, } 2017\end{array}$ \\
\hline & Detection of three arboreal mammal in complete darkness with thermal camera & $\begin{array}{l}\text { DJI Matrice 600Pro with } \\
\text { DJI Zenmuse XT V2.0 } \\
\text { FLIR uncooled thermal } \\
\text { sensor }\end{array}$ & Kays et al., 2019 \\
\hline \multirow{4}{*}{ Satellite } & Habitat heterogeneity and composition for 10 bird species with image processing & $\begin{array}{l}\text { Landsat Thematic Mapper } 5 \\
\text { (TM5) }\end{array}$ & $\begin{array}{l}\text { St-Louis et al., } \\
2014\end{array}$ \\
\hline & Whale counting with very high resolution satellite imagery & $\begin{array}{l}\text { WorldView2 with } 50 \mathrm{~cm} \\
\text { resolution }\end{array}$ & $\begin{array}{l}\text { Fretwell et al., } \\
2013\end{array}$ \\
\hline & $\begin{array}{l}\text { Monitor animal behaviour for a cattle enterprise by combining sensor data from } \\
\text { different modalities }\end{array}$ & SPOT-5 & $\begin{array}{l}\text { Handcock et al., } \\
2009\end{array}$ \\
\hline & $\begin{array}{l}\text { Manual counting of elephant seals with poor contrast against background in satellite } \\
\text { images }\end{array}$ & Geo-Eye satellite & $\begin{array}{l}\text { McMahon et al., } \\
2014\end{array}$ \\
\hline
\end{tabular}

programmed. It used solar powered battery operation to sustain two-way communication over the cellular network and image processing on Raspberry Pi (Nazir et al., 2017). Camera traps were used to understand scavenger behaviour at a Golden SnubNosed Monkey (Rhinopithecus roxellana) carcass (Huang et al., 2014). A solar powered battery powered satellite based remote 
monitoring system was developed to observe scavenger behaviour at a deer carcass. The image and video capture was motion activated using PIR sensors and image processing. The system live streamed the video data over the satellite link and enabled remote software configuration to the system (Nazir et al., 2016).

A 14 year program monitored the Jaguar population of 105 adult jaguars using camera traps in a camera trap study (Harmsen et al., 2017). This was the first study for population dynamics with a sufficiently large sample. The recognition of individuals was based on identification of the spot patterns. The study covered an area of $100 \mathrm{~km}^{2}$ with 19 locations for the camera traps (Harmsen et al., 2017).

Inspired by the decreasing costs, ease of camera trap studies, citizen science, and many successful larger scale camera trap studies (Burton et al., 2015; Wildlife Insights), a global network of camera traps for monitoring biodiversity is proposed in (Steenweg et al., 2017). Advances in camera trap technologies and their declining costs have enabled new surveys, but at times robust ecological studies require more than data collection (Burton et al., 2015).

\subsection{Bio-logging or Animal-borne Sensors}

It is advantageous for some studies to mount the sensing platform on the animal. The attachment of a sensor to an animal provides a much prolonged observation in the natural habitat and in different activity states. Thus these animal mounted cameras can be used to record activity from places which are otherwise not accessible. However a disadvantage is that this requires that the animal be captured, fitted with the biologger and then released.

Satellite telemetry for long-range bat movements was used in trials using Platform Terminal Transmitter (PTT) and collar on captive and free bats (Family Pteropodidae: Genus Pteropus) (Smith et al., 2011). The solar-powered PTT performance was found better than battery-powered ones for species with habitats favouring solar recharge. In future, PTT size and weight reduction will improve deployment lifetime (Smith et al., 2011).

These sensors can provide insights into animal behaviour difficult to observe in natural habitats. The foraging behaviour of New Caledonian (NC) crows (Corvus moneduloides) using tools was observed through fitting 19 birds with custom built videologgers to obtain 10 hours of video. These were first footages of manufacture and use of stick tools under completely natural foraging environment (Troscianko and Rutz, 2015). The camera was set to record automatically with two pre-set schedules and mounted for safe automatic detachment after 7 days (Troscianko and Rutz, 2015).

Animal borne video collars were used on 13 Tasmanian devil (Sarcophilus harrisii) to obtain 173 hours of recorded video to understand the activity and social interactions. This was the first study that found the importance of the devil in the ecosystem by manually analysing the captured video (Anderson et al., 2019). Use of high definition video loggers can provide more diverse information as demonstrated for yellow-eyed penguin study (Mattern et al., 2018). A camera logger was developed to record full HD video using a wide angle lens to understand the foraging behaviour of penguin. A total of 2 hours of H.264 video was recorded with video data written to a file every 3 mins. The light sensitivity of camera worked well to a depth of $70 \mathrm{~m}$ (Mattern et al., 2018). The size of animal-borne imaging and environmental data collection systems (AVEDs) is getting smaller and can thus be used on small animals. A harness was designed and used with dusky smoothhound sharks (Mustelus canis) for both laboratory and field conditions for understanding behaviour. The details of the harness are provided and mostly statistical techniques were applied for post processing. (O’Connell). A total of 6 sharks were captured for the field experiment and the swimming behaviour and predator-prey dynamics were analysed using statistical techniques (O’ Connell et al., 2020).

There has also been a trend to combine capture data through many sensors that can aid in better understanding of the monitored behaviour. The use of acceleration, gyroscope and time-depth recorder was validated for green turtle behaviour inference by recording 66 hours of video. The study used seven classifiers Linear Discriminant Analysis (LDA), Support Vector Machine (SVM), Classification and Regression Trees (CART), Random Forest (RF), Extreme Gradient Boosting (EGB), Voting Ensemble (VE) and Weighted Sums (WS) and found that WS gave the best results in automatic behavioural identification based on TDR, acceleration and gyroscopic data (Jeantet et al., 2020). 


\subsection{Drones}

Drones or Unmanned Aerial Vehicles (UAV) can carry on-board cameras to capture images and videos from inaccessible terrain such as monitoring behaviour of cliff nesting birds. Depending on the type of camera carried it can capture an image or video in any environment condition such as thermal image in complete darkness. Drones are increasingly being used due to the ease with which these can be flown over an area of interest to collect imagery. The mobile platform with on-board sensors can approach and monitor the intended animal on land, sea, or air. Such platforms may also be used in conjunction with the ground based sensors for data transfer.

With drones becoming a common tool for investigation and data collection, it has become possible to gather ecological data through drones (Hodgson et al., 2018). A review of drone use for conservation in protected areas with a classification of drones and type of imaging sensors that can be mounted on the drones is provided in (López and Mulero-Pázmány, 2019). Drones were used to get insights into mating dynamics and sex ratios of loggerhead sea turtles (Caretta caretta) through repeated surveys to determine the morphological differences such as tail length (Schofield et al., 2017). Use of drones to identify crocodile nests allowed a large area to be covered at lower cost, providing a methodology for nest surveying useful for similar studies for other species (Evans et al., 2016).

Some studies use drones with thermal cameras due to their inherent advantages. A preliminary study was carried out to determine if the thermal imagery would help establish the orangutan distribution and density, and showed that there is a correspondence between aerial and ground surveys (Wich et al., 2015). The full costs of drone studies should be carefully evaluated and in addition to the cost of equipment must consider the training for equipment operation (Wich et al., 2015). Drones were used mounted with thermal cameras for detection of orangutans (Pongo spp.) which make nests in the upper rainforest canopy. An altitude of $100 \mathrm{~m}$ provided the best balance between the image resolution, the transmission range, and coverage of area per flight (Dahlen and Traeholt, 2017). Drone mounted thermal cameras were used to scan large areas of rainforest canopy to detect high canopy species. It was suggested that better results could be obtained by combining, thermal, with flash photography or IR illumination. The disturbance to animals did not happen above 40m (Kays et al., 2019).

Whether the drones cause disturbance to animals is also an important factor to be considered. Disturbance to nesting birds with drones and providing considerations for reducing disturbance and enhanced image accuracy is described in (Mapes et al., 2020). Animal behaviour was studied using approaching drones at various angles and to assess impact of drones (Vas et al., 2014). There is also a trade-off in minimising disturbance and the ability to capture an image at a resolution and distance that aids analysis for animal counting etc. (Rush et al., 2018). Thermal imaging can capture temperature variations in and across bird nesting habitats. A counting application for breeding seabird colony of Black-backed Gulls (Larus fuscus) utilised image processing with a minimal disturbing drone for colony nesting seabirds (Rush et al., 2018). The semi-automated classification provided a high correlation with the manual count. Agisoft PhotoScan v1.2.4 software was used for image processing along with maximum likelihood classification. A low disturbance to birds was noticed with the drone flying at a height of $40 \mathrm{~m}$ (Rush et al., 2018). ). A good discussion on the future of drones in the field of ecology is provided by Crutsinger et al. (2016). Better and cheaper hardware, smart drone technology, and integration with mobile and cloud would increase use of drones in ecology (Crutsinger et al. (2016).

\subsection{Satellites}

The satellites cover the entire earth and satellite systems provide open access to satellite imagery and can be easily used to infer information for counting or movement of large-bodied animals. The resolution of the imagery has also been getting better and the satellites can cover larger areas of the earth. The use of satellite imaging helps to identify target animal and population and is generally best suited for applications with a homogeneous background as in snowy terrain or sea water for better contrast facilitating discrimination of the target animal.

The satellite data is now more widespread and easily available for ecological research (Kwok. 2018). The image resolution has also improved which makes animal identification better and also more information can be extracted (Wang et al., 2010). The availability of satellite data can be very useful for ecology studies through the use of open source data and tools (Kwok, 2018). A review of remote sensing for monitoring biodiversity and conservation covering satellites, LIDAR and Global Information System (GIS) is provided by Wang et al. (2010). Raw, unclassified remote satellite imagery was used to model avian biodiversity (St-Louis et al., 2014). The measures from satellite remote sensed data, habitat heterogeneity and habitat composition were compared for bird species diversity. Raw digital numbers from satellite images were converted to surface 
reflectance as a two stage pre-processing (St-Louis et al., 2014). Image texture was used with satellite based techniques for determining biological diversity (St-Louis et al., 2014).

A study combining GPS and Wireless Sensor Network (WSN) data with satellite images for monitoring animal behaviour is described in (Handcock et al., 2009). It used a combination of mobile sensors with static nodes for data backhaul, with the data correlated with satellite images (Handcock et al., 2009).

The elephant seals were manually counted in satellite imagery and validated against the ground truth information with a high correlation (McMahon et al., 2014). A satellite image of the study area was acquired with a pixel size of $0.5 \mathrm{~m}$ and panchromatic image was used. The study demonstrated the possibility of successful count of animals with poor contrast against the background which is useful for other such studies (McMahon et al., 2014).

\section{ADVANCED IMAGE PROCESSING TECHNIQUES}

This section provides details of the studies that use advanced image processing techniques to answer the various research questions and are grouped as species recognition, animal counting, animal tracking, behaviour monitoring and biodiversity.

\subsection{Species Recognition}

Species recognition or classifying a given image into one of the species categories is the most common task in deep learning and ecological studies. This section describes how the machine learning models can be used for image based species identification (Wäldchen and Mäder, 2018). Similarity scores based on a three-dimensional model through custom software were calculated to identify a tiger based on its skin stripes (Hiby et al., 2009). This could be a foundation to develop similar software for other uniquely striped species (Hiby et al., 2009).

An automated method was developed for automatic classification of Cichlid fish (500 to 800) species to differentiate based on colour, stripe, shape of jaw, and body (Joo at al., 2013). The features were automatically extracted and were classified using Support Vector Machine (SVM) and Random Forests (Joo et al., 2013). Arthropod species identification was performed using stacked random forests and dictionary learning with boosting (Dietterich, 2009). An algorithm was designed for smart phone for studying Desert tortoise and Mohave Ground Squirrel animal populations in Mohave Desert (Wilber et al., 2013). The algorithm was based on Local Binary patterns (LBP) and Scale-invariant Feature Transform (SIFT) for extracting features and SVM for classification. The data was collected by luring the animals into the field of view.

An automated species recognition system based on dense SIFT descriptors and cell structured LBP (cLBP) features followed by classification based on linear Support Vector Machine (SVM) is proposed in (Yu et al., 2013). The study considered over 7000 images of 18 species from two sites and an accuracy of $82 \%$ results were obtained. The dataset was unbalanced due to which species with few representations were removed. To get clear data despite changes to illumination conditions, the animals were manually cropped from the sequence. The species recognition was based on an improvement to Sparse Spatial Pyramid Matching (ScSPM) implementation.

A first study involving CNN algorithm was used for wild animal species recognition by segmenting the camera trap motion activated images region of interest (ROI) using graph-cut algorithm (Chen et al., 2014). The study annotated a camera trap dataset of 20 species and compared the proposed techniques against traditional bag of visual words. The performance of CNN for species recognition was only 38.31\% accurate due to limited training data (Chen et al., 2014).

A pre-trained CNN (ResNet-18) model was trained with a collection of images and then tested on unseen datasets with 97.6\% accuracy as the best achieved for images from various camera trap studies (Tabak et al., 2019).

A fully automated pipeline comprised of face detection, tracking and recognition of wild chimpanzees (P. troglodytes verus) from video records with over 30 years of video data (schofield et al., 2019). The evaluation of results between human expert annotators and the automated pipeline showed that on a frame level identity classification task, expert human scored $42 \%$ in about 55 min whereas the software achieved $84 \%$ in 60 ms. Interestingly, the study did not constrain the video data in any way but the actual raw video with little pre-processing was used (schofield et al., 2019). 
The identification of animal species in camera trap images with model training on images classified by citizen scientists at Zooinverse (zooinverse.org) demonstrated that enlisting citizen scientists this can substantially cut the effort required by researchers (Willi et al., 2018). They also investigated how to process small datasets that are difficult to model and to investigate the accuracy of CNNs in classifying image data by comparing transfer learning and fully trained models. Classification accuracy was found to be low for animals with less representation in the dataset (Willi et al., 2018).

\subsection{Population Abundance Monitoring/Animal Counting/Density Estimation}

Population estimates can answer relies on accurate species recognition to answer many research questions. Animal counting can provide insights into population health and the ecosystem (Valletta et al., 2017). A study performed in-situ monitoring of coral reef fish using multi factorial uncertainty assessment for monitoring population abundance (Beauxis-Aussalet and Hardman, 2015). The study aims to identify uncertainty factors in video monitoring systems (Beauxis-Aussalet and Hardman, 2015).

An efficient means for survey design in conservation planning based on spectral signal of remotely sensed images (Mellin et al., 2012). Distance-based partial redundancy analysis and hierarchical linear models were used to calculate an index for univariate and multivariate spatial heterogeneity. Both in-situ digital photographs and remote sensed satellite imagery were used.

Counting of Wildebeest in thousands of aerial photos can be a very time consuming process (Valletta et al., 2017). Principal Component Analysis (PCA) and Random Forest as classifier were used to obtain a good training and predictive performance (Valletta et al., 2017).

Automatic localization and animal counting with conservation drones utilising object detection techniques offers advantages over manual operation on foot or from aerial recordings (Gemert at al., 2014). Object detection for animal abundance monitoring drones showed promising results with three techniques, exemplar Deformable Part-based Model (DPM), colorDPM and SVM (Gemert et al., 2014). In another study with drones it similarly concluded through semi-automated counting techniques using SVM, in drone imagery for sea birds to provide more accurate results compared to manual counting (Hodgson et al., 2018).

The automated analysis of fish abundance using CNN on underwater videos showed that the performance of the model was 7.1\% better than the human expert (Ditria et al., 2020). Deep learning was applied for automatic identification and counting of animals from a camera trap image database containing 3.2 million images from Snapshot Serengeti dataset, using deep Convolutional Neural Networks (CNN) (Norouzzadeh, 2018). The accuracy reported for animal identification was 93.8\% highlighting enormous savings in time and effort against manual identification and counting of animal species. The events considered were where only a single species was present. $75 \%$ of the capture events were devoid of animals and also the dataset was very unbalanced in that there was uneven representation of animal species. The predefined architectures such as AlexNet, GoogleNet were used and accuracies for different architectures were compared (Norouzzadeh et al., 2018). The attributes for the animal image, that is, standing, resting, moving, interacting, and whether with young were also inferred to around $75 \%$ accuracy (Norouzzadeh, 2018).

The image processing techniques were used to identify and count whales in satellite images (Fretwell et al., 2014). They used both supervised and unsupervised classification to identify and count whale images (Fretwell et al., 2014). The abundance and distribution of airborne animals can be measured with radar. This has many challenges and requires interpretation and analysis of radar data (Stepanian et al., 2014). Image processing on radar images comprising simplified steps to recognise animals can work by setting a background threshold to segregate animals, identify contiguous pixels, and then label them (Stepanian at el., 2014). The weather surveillance radar can provide the biomass movement of birds and bats over large area continuously and that can help in migratory studies (Schmaljohann, 2020).

In this first study to count whales, satellite images were used to identify Southern right whales (Eubalaena australis). The satellite image represented $113 \mathrm{~km}^{2}$ area of a location where whales abound (Fretwell et al., 2014). ENV15 and ArcGIS software was used with maximum likelihood supervised and unsupervised (isoData and k-means) classification. It was found that a simple thresholding technique with the unsupervised classification provided the best results. 
Drones were used to capture images of olive ridley turtles (Lepidochelys olivacea) that were then analysed using CNN for and demonstrated training on relatively small $(\mathrm{N}=944)$ images (Gray et al., 2018). The manual validation requirement was shown to be reduced substantially. The CNN model comprised of 4 convolutional layers and the images were divided into 100x100 pixel windows for training the model.

\subsection{Animal Tracking}

Animal tracking using video to determine the pose and position is the first step for understanding animal behaviour (Branson, 2014). Automated image-based tracking makes it possible to remotely understand the animal behaviour. Video based tracking has greater potential. Image based tracking consists of image acquisition, detecting an individual and pose to create trajectories in succeeding images, and behavioural data analysis. Multiple cameras are needed for tracking in 3D environments (Dell et al., 2014).

Tracking for terrestrial animals can be done globally using satellite tags and cellular networks which are also helped enormously by combining the information from many sensors. This can also be used to deduce information about animal's interactions and multi-target tracking (Kays et al., 2015). Tracking through computer vision can automate much of laborious work in animal tracking. The results are provided for ants, bees and monkeys to understand the social behaviour and interactions (Balch et al., 2006). Initially the system could track only one monkey but was later extended to simultaneous tracking of 60 monkeys in a 30m x30 m area. Multitarget tracking algorithms have also been described (Balch et al., 2006).

Animal trajectory tracking to find the degree of motion due to pathology (gastric ulcer) is reported in (Koniar et al., 2016) using two background subtraction methods based on thresholds and colour matching. The method provided an inexpensive alternative to Radio Frequency (RF) methods. The methods were tested on caged guinea pigs under various lighting conditions. Tracking tags can have multiple sensors such as accelerometers, cameras, heart rate, temperature etc. Tracking of multiple animals leads to new insights into ecology (Kays et al., 2015). An id Tracker system is described that can track unmarked individuals by recognizing them repeatedly, and can work with fish, flies, ants and mice (Branson, 2014).

\subsection{Animal Behaviour/Activity Monitoring}

Automatic labelling of the animal behaviour can be performed using computer vision, and can provide better and consistent results compared to manual labelling (Balch et al., 2006). A review of camera trap studies for animal behaviour is provided in (Caravaggi et al., 2017) covering the areas of anthropogenic impacts on behaviour, use of behaviour patterns, and considering behavioural responses in management. Animal tracking can provide insights into the animal behaviour (Dell et al., 2014). Unsupervised methods can help ecologists as the manual behaviour labelling can be avoided (Dell et al., 2014).

A review of machine learning applications for analysis of complex animal behavioural data is provided together with three cases studies. In one case study, a total of 1000 jackdaws across three colonies were fitted with Passive Integrated Transponder (PIT) and data was collected by recording visit of each bird to a bird feeder. These timestamps were then used to infer the social networks within the group by applying Gaussian Mixture Model GMM to cluster the data (Valletta et al., 2017).

Drones could reach quite close without registering a reaction in most cases from mallards (Anas platyrhynchos), wild flamingos (Phoenicopterus roseus), and common greenshanks (Tringa nebularia) (Vas et al., 2014). By integrating a motion activated speaker system, the animal behaviour to anthropogenic disturbances was studied in (Suraci et al., 2016). The camera traps were used for capturing the behaviour through their Automated Behavioural Response system (Suraci et al., 2016). Similarly, data from multiple sensors were combined considering contextual, spatial and temporal constraints for better behaviour understanding (Handcock et al., 2009).

Machine learning techniques, such as, decision trees/rules, neural networks; support vector machines and genetic programming were applied to the problem of spatial distribution of southern brown bandicoot (Isoodon obesulus) with poor data. Decision trees and genetic programming gave better results in the context of the studied problem (Shan, 2006). Data from 344 sites was used which was unbalanced and also had some missing values. It was concluded that the dataset used proved highly resistant to analysis (Shan, 2006).

\subsection{Biodiversity}

Biodiversity and conservation policies can be guided through habitat models on remote sensed data (St-Louis et al., 2014). A review of remote satellite sensing for biodiversity is provided in (Wang et al., 2010). The biodiversity in fish species over a reef 
was established using spatial information in digital images signifying the importance of unprocessed remotely sensed images. It was the first multi-scale study to derive fish biodiversity patterns from a combination of satellite and camera images. Still images were used from the underwater video surveys. The proposed method of mean information gain (MIG) was used to measure the fish community structure (Mellin et al., 2012). The Tropical Ecology Assessment and monitoring (TEAM) network uses methods to collect near real-time data on biodiversity in 17 sites across three continents (Wildlife Insights).

Camera traps can be used not only for biodiversity measurement but to infer the underlying causes (Steenweg et al., 2017). These remote cameras can provide estimates of animal populations to help monitor biodiversity at a global scale and inform questions relating to conservation policy. A global ecological monitoring similar to weather monitoring needs to be deployed with interconnected ecological sensors. The suggested measures to overcome barriers to setup regional camera trap networks were standardisation of protocols, automating image processing, sharing and access to data (Steenweg et al., 2017).

\section{FUTURE DIRECTIONS AND CHALLENGES}

The recent technological innovations have started to transform the field of ecology similar to other sciences. Following technological breakthroughs would drive the innovations in the field of ecology.

\subsection{Prolonged Image Acquisition Operations}

The provision of low power devices with more processing power and longer battery life together with reduced sizes will open and advance many ways so far unknown or limited for use of such devices. Although camera traps are usually employed to capture images but videos provide more detailed information (Swinnen et al., 2014). With the miniaturization of camera traps and animal-borne sensors, it will become feasible to continuously monitor with a high resolution video capture for an event of interest rather than only recording video at pre-set time or using time lapse images.

Renewable energy sources have already been used in camera traps for ensuring continuous and autonomous operation (Nazir et al., 2016) and animal borne loggers (Smith et al., 2011). There are many commercial applications being developed to prolong system operational time using solar panels. A battery operated solar powered system for continuous operation was deployed in the field to backhaul live image and video captured off-site over a satellite link (Nazir et al., 2016). Similarly better battery technologies and solar powered drone will enable large scale ecological surveys.

With an enhanced operating time for the image capture devices it would become possible to run the image analysis on the acquisition device (drone or a camera trap) such as species recognition and to communicate back the result of the analysis rather than waiting for the data to be collected and then analysed.

\subsection{Near real-time Information}

The captured data is usually acquired through physical access to the device as in the case of camera traps. However, the communications technologies with a wide choice of communications range, coverage, cost and power requirements have made it possible to acquire the data in near real-time or periodic basis from the field site.

Real-time data acquisition from tagged animals has transformed the way information is utilised (Kays et al., 2015; van der Wal et al., 2015). Technology would soon enable real-time collection of information from the wild (Norouzzadeh et al., 2018).The remote monitoring system provided information backhaul of data, images and videos from the field with the possibility to connect to the live video feed (Nazir et al., 2016).Camera traps are available that can transmit data about the state (e.g. battery voltage) and captured images and videos directly. However, in future there would be increased reliance on being able to change the system parameters and algorithms (Nazir et al., 2017) to meet the change in weather, study requirements or to capture relevant information, such as video, on detecting an animal presence (Nazir et al., 2016). Similarly drones can provide live video transmission; the distance from drone to controller was 2.5km (Dahlen and Traeholt, 2017).

By integrating the sensors and cameras deployed all across the globe, comprising satellite, camera traps, animal-borne video loggers, and drones, it will soon be possible to monitor the wildlife in real time for animal conservation.

\subsection{Drones and Robotics}

Robots are important in studies where human presence is not advisable due to the extreme weather, effect on animal behaviour, dangers from animals, or hostile environment such as deserts, oceans etc. Algorithms from robotics community can 
help researchers to track and understand the social interactions between social animals (Balch et al., 2006). Drone technology has the advantage that it can be used for image, video and sensor data capture from inaccessible location quite easily.

Robot is an autonomous platform with locomotion, positioning and sensing capability (Grémillet et al., 2012). These may be deployed for data acquisition from otherwise inaccessible locations such as subsea terrain, deserts, and caves. The robots in ecology could change biodiversity sampling, population dynamics, etc. Robots could be released amongst biological creatures to understand behaviour (Grémillet et al., 2012).

Drones have many advantages but some challenges need to be overcome that are difficulty in detecting animals with lesser difference from ambient temperature, difficulty in identifying animals at night, and logistical constraints on import and performance (Kays et al., 2019).

Advances in technology will enable remote deployment of multiple agents to a location and then remote controlled or autonomous navigation to another location of interest. This could pave the way to detect and monitor the rare and elusive animals. Similarly with underwater camera mounted robots employing various image capture techniques will make it easier to acquire images.

\subsection{Big Data}

Big data is commonly defined to have three characteristics as volume, variety, and velocity of data. Big data can provide valuable insights; however the data deluge could create disdain or lack of interest (Peters, 2014). The use of automatic image capture techniques for example, camera traps, can generate very large data sets (Caravaggi et al., 2017). Camera trap studies could generate thousands or millions of images (Yu et al., 2013).

Extracting information from camera trap images is an expensive and laborious task (Norouzzadeh et al., 2018). The ability to accurately and inexpensively collect image data through camera trap images and applying deep learning techniques on it for identification and species count could transform ecology into big data sciences (Norouzzadeh et al., 2018). Consider for example supervised machine learning techniques that require labelled data. Finding labelled data is a challenge (Weinstein, 2017). With large scale datasets requiring persons with domain knowledge to label data is a difficult task.

Although the problems in ecology are similar to well-studied problems such as object detection, optimization etc. but the solutions need to be modified for their application (Dietterich, 2009). For example, predicting 5000 species jointly poses challenges as more than 12 million interactions could exist between pairs of species, infeasible for estimation even from large datasets (Dietterich, 2009).

Millions of images from camera trap and other studies are difficult for the researchers to process. The identification of animal species in large camera trap datasets can be aided by combining citizen scientists and CNNs that can provide good results in less time (Willi et al., 2018).

\subsection{Advanced Imaging Techniques}

The significance of thermal camera is a possibility now but as yet advanced image processing techniques have not been applied to the thermal images for species identification etc. Animals can be detected in poor contrast and with better satellite imagery (McMahon et al., 2014), it might be possible to get even better results for elusive species of small size. Better image resolution and zooming with thermal cameras would make it possible to detect and identify small species (Dahlen and Traeholt, 2018). With satellites, better resolution images with many imaging modalities are available and accessible.

There are many innovations in the imaging arena that would soon be available for widespread adoption, such as, better video compression standards, H.265 that can compress to 50\% more compared to the same quality of H.264. Thermal cameras have opened new insights into hard to observe animal but similarly 360 degree, 3D and hyperspectral imaging would provide new insights into information henceforth inaccessible.

Better results have been demonstrated by combining the data from more than one sensor (Kays et al., 2015; Nazir et al., 2017). This would become more common and beneficial through opportunities made possible with decreased costs, miniaturisation, and storage. 


\subsection{Cloud and Edge Computing}

Cloud technology provides a platform that makes available an unlimited storage, processing and networking capability at much reduced costs and at a global scale. The major cloud vendors are Amazon, Google and Microsoft with each providing pretrained machine learning models that are readily available to be used. Based on the current trajectory of cloud adoption, and the ever increasing requirement of ecological applications of data processing and storage, it provides an ideal platform for storage and processing of collections of millions of images. The potential of suitably trained deep learning models is promising for real-time identification and analysis of ecological data.

With the availability of cloud resources and improved communications technologies, the data can be uploaded from the field devices (camera traps, drones etc.) directly to the cloud platform, which can then be processed and results disseminated to concerned stakeholders in near real-time. The only hindrance if at all to establish such a data and processing pipeline is the communications technology from the field to the cloud where again better alternatives are becoming available.

Edge computing is an enabler for many technologies and provides a quicker response time by making the storage and processing capabilities available closer to the low power devices. Thus the low power devices can upload the images and video for further heavy processing. This would potentially affect the ecological research in a big way by providing rapid response, increased technology adoption and informed research.

\subsection{Deep Learning}

Deep learning has already made it possible to have results similar or better to those of expert humans in animal classification at the fraction of cost and time. The use of deep learning approaches will not only increase in species recognition (Waldchen and Mader, 2018) but also for the other data processing challenges facing the ecologists. Using transfer learning, the heavier data processing of training a model can be done on a powerful computer and the trained model can be used for prediction.

It is now possible to run deep learning with TensorFlow Lite and TinyML on the embedded platforms or the low end devices. The field of deep learning is also progressing and better models are available. The animal classification requires labelled data that is an onerous task for the supervised learning mostly used for classification. The other branch of deep learning, that is unsupervised learning and reinforcement learning is also useful but have not been exploited much yet. Deep learning models can also automatically provide contextual and semantic information as textual description based on the information in the image.

Good results with clean data have been achieved for image classification with deep learning. A challenge is to develop models that would work well on challenging sets of images with poor resolution and illumination for animal biometrics (Schofiled et al., 2019).

\subsection{Data Sharing, Dissemination and Collaboration}

Long Term Ecological Research (LTER) project (https://ternet.edu/site/) has been running since 1980 and currently has 28 sites with the datasets available online. Such data sharing can progress the research frontiers. There is in general a need for data sharing and dissemination beyond the lifetime of the project. Data management and knowledge discovery for ecological informatics provides five case studies covering ecological informatics evolution to meet the challenges in ecology besides describing relevant and important topics in data management (Recknagel and Michener 2018).

Making a device hardware or software open source can help the research community to take the work forward and improve on the earlier work. Open source device such as camera trap (Nazir et al., 2017), and an OpenDropOff unit for animal-borne devices (Rafiq et al., 2019) can benefit the whole community of researchers.

There is a need for interdisciplinary collaboration to help tackle the problems and issues. The need of collaboration between data scientists and ecologists would help tackle the enormous amount of data that gets collected but never gets analysed due to limited resources (Farley et al., 2018). Similar collaborations between ecologists and computer scientists and remote sensing specialists would aid the interpretation of satellite imagery. 


\section{CONCLUSION}

The ease of deployment of digital devices to gather ecological data has resulted in a data deluge, emphasizing the need for use of technology not only to gather data but to process and interpret it. This paper provides information on how the image acquisition and processing technologies are facilitating and transforming the ecological methods and processes. We describe the image modalities and various image acquisition technologies. We also provide coverage of how deep learning techniques are aiding to help recognize and categorize animal autonomously. These provide much improved results for computer vision compared to low level techniques employed earlier.

Ecological studies have seen an emergence of deep learning model utilisation. However, much more needs to be done before use of these technologies becomes commonplace and can be successfully applied to ecological studies. In future the complete data pipeline from sensing and acquisition in the field to data analysis and inferences would become automated enabled and powered by the cloud technology.

There is greater need for computer scientists to collaborate as ecology moves to become a data-driven science (Dietterich, 2009). The collaboration between ecologists and computer vision researchers are important and can benefit areas such as automation of image analysis (Weinstein, 2017). However, these collaborations are generally restricted to a project only but have potential to be more widespread. The ecological challenges can be addressed in collaboration between computer scientists and ecologists to develop customised computational tools (Dietterich, 2009).

At a broader scale, considering the importance of ecology for climate, food security and environment, there is an emergent need for collaboration between the various research bodies to increase awareness, monitor, and effect improvement. Such collaborations bring together expertise in specialist domains that would spur new insights and understanding for the sustenance and betterment of all life forms on the planet.

\section{Funding:}

This research did not receive any specific grant from funding agencies in the public, commercial, or not-for-profit sectors.

\section{References}

G. E. Andersen, H. W. McGregor, C. N. Johnson, M. E. Jones, “Activity and social interactions in a wide ranging specialist scavenger, the Tasmanian devil (Sarcophilus harrisii), revealed by animal borne video collars” PLoS ONE 15(3): e0230216. https://doi.org/10.1371/journal. pone.0230216

T. Balch, F. Dellaert, A. Feldman, A. Guillory, C. L. Isbell Jr., Z. Khan, S. C. Pratt, A. N. Stein and Hank Wilde, "How multirobot systems research will accelerate our understanding of social animal behaviour," Proceedings of the IEEE, vol. 94, no. 7, pp. 1445-1463, Jul. 2006.

E. Beauxis-Aussalet and L. Hardman, "Multifactorial uncertainty assessment for monitoring population abundance using computer vision,” 2015 IEEE International Conference on Data Science and Advanced Analytics (DSAA), Paris, 2015, pp. 1-10, DOI: 10.1109/DSAA.2015.7344879.

K. Branson, "Distinguishing seemingly indistinguishable animals with computer vision," Nature Methods, vol.11, no.7, pp. 721-722, Jul. 2014.

C. Burton, E. Neilson, D. Moreira, A. Ladle, R. Steenweg, J. T. Fisher, E. Bayne and S. Boutin, "Wildlife camera trapping: a review and recommendations for linking surveys to ecological processes,” Journal of Applied Ecology, 52, pp. 675-685, 2015, DOI: 10.1111/1365-2664.12432.

A. Caravaggi, P. B. Banks, A. C. Burton, C. M. V. Finlay, P. Haswell, M. Hayward, J. M. Rowcliffe and M. D. Wood, “A review of camera trapping for conservation behaviour research,” Remote Sensing in Ecology and Conservation, 2017.

G. Chen, T. X. Han, Z. He, R. Kays and T. Forrester, "Deep Convolutional Neural Network Based Species Recognition for Wild Animal Monitoring,” 2014 IEEE International Conference on Image Processing (ICIP), Paris, 2014, pp. 858-862, DOI: 10.1109/ICIP.2014.7025172. 
S. Christin, É. Hervet, and N. Lecomte, “Applications for deep learning in ecology,” Methods in Ecology and Evolution, vol. 10, no. 10, 2019. DOI:10.1111/2041-210X.13256.

G. M. Crutsinger, J. Short, and R. Sollenberger, "The future of UAVs in ecology: an insider perspective from the Silicon Valley drone industry” J. Unmanned Veh. Syst. 4: 161-168 (2016) dx.doi.org/10.1139/juvs-2016-0008

B. Dahlen and C. Traeholt, "Successful aerial survey using thermal camera to detect wild orangutans in a fragmented landscape”

A. I. Dell, J. A. Bender, K. Branson, I. D. Couzin, G. G. de Polavieja, L.P.J.J. Noldus, A. Perez-Escudero, P. Perona, A. D. Straw, M. Wikelski, and U. Brose, “Automated image-based tracking and its application in ecology” Trends in Ecology \& Evolution, July 2014, Vol. 29, No. 7

P. Dickinson, R. Freeman, S. Patrick and S. Lawson, "Autonomous monitoring of cliff nesting seabirds using computer vision,” International Workshop on Distributed Sensing and Collective Intelligence in Biodiversity Monitoring, Dec. 2008, Amsterdam.

T. G. Dietterich, "Machine learning in ecosystem informatics and sustainability," Proceedings of the Twenty-First International Joint Conference on Artificial Intelligence (IJCAI-09), pp. 8-13, Jul. 2009.

E. M. Ditria, S. Lopez-Marcano, M. Sievers, E. L. Jinks, C. J. Brown and R.M. Connolly, “Automating the Analysis of Fish Abundance Using Object Detection: Optimizing Animal Ecology With Deep Learning” Front. Mar. Sci. 7:429. doi: 10.3389/fmars.2020.00429

L. J. Evans, T. H. Jones, K. Pang, S. Saimin and B. Goossens, "Spatial ecology of estuarine crocodile (Crocodylus porosus) nesting in a fragmented landscape,” Sensors, 16, 1527, 2016, DOI: 10.3390/s16091527.

P. T. Fretwell, I. J. Staniland, J. Forcada, "Whales from Space: Counting Southern Right Whales by Satellite”, PLOS ONE, vol. 9, issue 2, 2014.

S. S. Farley, A. Dawson, S. J. Goring, and J. W. Williams, "Situating Ecology as a Big-Data Science: Current Advances, Challenges, and Solutions,” BioScience 68: 563-576. (c) The Author(s) 2018, doi:10.1093/biosci/biy068

P. C. Gray, A. B. Fleishman, D. J. Klein, M. W. McKown, V. S. Bézy, K. J. Lohmann, D. W. Johnston, “A convolutional neural network for detecting sea turtles in drone imagery” Methods Ecol Evol. 2019;10:345-355.

J. C. van Gemert, C. R. Verschoor, P. Mettes, K. Epema, L. P. Koh, and S. Wich (2015). "Nature conservation drones for automatic localization and counting of animals,” In: L. Agapito, M. Bronstein, \& C. Rother (Eds.), Computer Vision ECCV 2014 Workshops: Zurich, Switzerland, Sep. 6-7 and 12, 2014: proceedings (vol. 1, pp. 255-270). (Lecture Notes in Computer Science, vol. 8925). Cham: Springer. DOI:10.1007/978-3-319-16178-5_17

A. S. Glen, S. Cockburn, M. Nichols, J. Ekanayake and B. Warburton, "Optimising Camera Traps for Monitoring Small Mammals,” PLOS ONE, vol. 8, no. 6, June 2013, e67940. DOI:10.1371/journal.pone.0067940

D. Grémillet, W. Puech, V. Garçon, T. Boulinier and Y. L. Maho, "Robots in ecology : welcome to the machine," Open Journal of Ecology, vol. 2, no. 2, pp. 49-57, 2012, DOI:10.4236/oje.2012.22006.

R. N. Handcock, D. L. Swain, G. J. Bishop-Hurley, K. P. Patison, T. Wark, P. Valencia, P. Corke and C. J. O’Neill "Monitoring animal behaviour and environmental interactions using wireless sensor networks, GPS collars and satellite remote sensing,” Sensors, 9, pp. 3586-3603, 2009. DOI:10.3390/s90503586

B. J. Harmsen, R. J. Foster, E. Sanchez, C. E. Gutierrez-Gonzalez, S. C. Silver, L. E. T. Ostro, M. J. Kelly6, E. Kay, and H. Quigley, "Long term monitoring of jaguars in the Cockscomb Basin Wildlife Sanctuary, Belize; Implications for camera trap studies of carnivores”, PLoS ONE 12(6): e0179505. https://doi.org/10.1371/journal.pone.0179505 
L. Hiby, P. Lovell, N. Patil, N. S. Kumar, A. M. Gopalaswamy, and K. U. Karanth, "A tiger cannot change its stripes: using a three-dimensional model to match images of living tigers and tiger skins,” Biol. Lett. (2009) 5, pp. 383-386.

J. C. Hodgson, R. Mott, S. M. Baylis, T. T. Pham, S. Wotherspoon, A. D. Kilpatrick, R. R. Segaran, I. Reid, A. Terauds, and L. P. Koh, "Drones count wildlife more accurately and precisely than humans," Methods in Ecology and Evolution, 9, pp. 1160-1167, 2018. DOI: 10.1111/2041-210X.12974.

Z. Huang, X. Qi, P. A. Garber, T. Jin, S. Guo, S. Li and B. Li, "The use of camera traps to identify the set of scavengers preying on the carcass of a Golden Snub-nosed monkey (Rhinopithecus roxellana),” PLoS ONE, , vol. 9, no. 2, Feb. 2014, e87318. DOI:10.1371/journal.pone.0087318

L. Jeantet et al. 2020 Behavioural inference from signal processing using animal-borne multi-sensor loggers: a novel solution to extend the knowledge of sea turtle ecology. R. Soc. Open Sci. 7: 200139. http://dx.doi.org/10.1098/rsos.200139

D. Joo, Y. Kwan, J. Song, C. Pinho, J. Hey and Y. Won, "Identification of cichlid fishes from Lake Malawi using computer vision,” PLOS ONE, vol. 8, no. 10, Oct. 2013, e77686.

R. Kays, M. C. Crofoot, W. Jetz and M. Wikelski, “Terrestrial animal tracking as an eye on life and planet” Ecology, vol. 348, no. 6240, 2015. DOI:10.1126/science.aaa2478

R. Kays, J. Sheppard, K. Mclean, C. Welch, C. Paunescu, V. Wang, G. Kravit and M. Crofoot, "Hot monkey, cold reality: surveying rainforest canopy mammals using drone-mounted thermal infrared sensors" International Journal of Remote Sensing, 40:2, 407-419, DOI: 10.1080/01431161.2018.1523580

D. Koniar, L. Hargas, Z. Loncová, F. Duchon, P. Beno, "Machine vision application in animal trajectory tracking," Computer Methods and Programs in Biomedicine, 127 (2016) pp. 258-272, DOI:10.1016/j.cmpb.2015.12.009.

A. Krizhevsky, I. Sutskever, G. Hinton, "ImageNet classification with deep convolutional neural networks," Communications of the ACM, vol. 60, no. 6, pp.84-90, May 2017.

T. E. Kucera and R. H. Barrett “A History of Camera Trapping,” In: A. F. O’Connell, J. D. Nichols, K. U. Karanth (eds) Camera Traps in Animal Ecology. Springer, Tokyo. 2011. DOI:10.1007/978-4-431-99495-4_2

R. Kwok, “Ecology’s remote-sensing revolution,” Nature, vol. 556, pp. 137-138, Apr. 2018.

J. J. López and M. Mulero-Pázmány, “Drones for conservation in protected areas: Present and future,” Drones, 3, 10, 2019, DOI:10.3390/drones3010010

LTER: Long Term Ecological Research: https://ternet.edu/vision-mission/

K. L. Mapes, N. G. Pricope, J. B. Baxley, L. E. Schaale, and R. M. Danner, "Thermal Imaging of Beach-Nesting Bird Habitat with Unmanned Aerial Vehicles: Considerations for Reducing Disturbance and Enhanced Image Accuracy” Drones 2020, 4, 12; doi:10.3390/drones4020012

T. Mattern, M. D. McPherson, U. Ellenberg, Y. van Heezik and P. J. Seddon, "High definition video loggers provide new insights into behaviour, physiology, and the oceanic habitat of a marine predator, the yellow-eyed penguin” PeerJ 6:e5459; DOI 10.7717/peerj.5459

C. R. McMahon, H. Howe, J. van den Hoff, R. Alderman, H. Brolsma, M. A. Hindell, "Satellites, the All-Seeing Eyes in the Sky: Counting Elephant Seals from Space”, PLoS ONE 9(3): e92613. doi:10.1371/journal.pone.0092613.

P. D. Meek and A. Pittet, "User-based design specifications for the ultimate camera trap for wildllife research," Wildlife Research vol. 39, no. 8, pp. 649-660, Dec. 2012. DOI:10.1071/WR12138.

C. Mellin, L. Parrott, S. Andréfouët, C. J. A. Bradshaw, M. A. MacNeil and M. J. Caley, "Multi-scale marine biodiversity patterns inferred efficiently from habitat image processing,” Ecological Applications, vol. 22, no. 3, pp. 792-803, 2012 
S. Nazir, G. Fairhurst, and F. Verdicchio, “WiSE - a satellite based system for remote monitoring,” Int. J. Satell. Commun. Network, 35, pp. 201-214, 2016. DOI: 10.1002/sat.1176.

S. Nazir, S. Newey, R. J. Irvine, F. Verdicchio, P. Davidson, G. Fairhurst, and R. van der Wal, "WiseEye: Next Generation Expandable and Programmable Camera Trap Platform for Wildlife Research,” , PloS ONE, vol. 12 , no. 1 , e0169758 DOI:10.1371/journal.pone.0169758

S. Nazir, S. Patel and D. Patel, "Assessing Hyper Parameter Optimization and Speed Up for Convolutional Neural Networks," International Journal of Artificial Intelligence and Machine Learning (IJAIML) vol. 10, no. 2, 2020. DOI: 10.4018/IJAIML.2020070101

S. Newey, P. Davidson, S. Nazir, G. Fairhurst, F. Verdicchio, R. J. Irvine, R. van der Wal, "Limitations of recreational camera traps for wildlife management and conservation research: A practitioner’s perspective,” Ambio 2015, 44 (Suppl. 4):S624-S635, DOI 10.1007/s13280-015-0713-1.

M. S. Norouzzadeh, A. Nguyen, M. Kosmala, A. Swanson, M. Palmer, C. Packer and J. Clune, "Automatically identifying, counting, and describing wild animals in camera-trap images with deep learning,” PNAS, 115 (25), Jun. 2018, DOI:10.1073/pnas.1719367115

O’Brien, T.G. (2011) “Abundance, density and relative abundance: a conceptual framework,” Camera Traps in Animal Ecology (eds A.F. O’Connell, J.D. Nichols \& K.U. Karanth), pp. 71-96. Springer, New York city, New York, USA.

C. P. O’Connell, C. Collatos , N. D. Picha and P. He, “A new animal-borne imaging system for studying the behavioral ecology of small sharks: laboratory and field evaluations,” Marine and Freshwater Behaviour and Physiology, 53:3, 131150, 2020, DOI:10.1080/10236244.2020.1782753

K. Rafiq, R. G. Appleby, J. P. Edgar, N. R. Jordan, C. E. Dexter, D. N. Jones, A. R. F. Blacker, and M. Cochrane, “OpenDropOff: An open-source, low-cost drop-off unit for animal-borne devices” Methods Ecol Evol. 2019;10:15171522.

F. Recknagel, W. K. Michener, Eds, “Ecological Informatics-Data Management and Knowledge Discovery”, Springer 2018.

F. Rovero, L. Collett, S. Ricci, E. Martin and D. Spitale, "Distribution, occupancy, and habitat associations of the gray-faced sengi (Rhynchocyon udzungwensis) as revealed by camera traps," Journal of Mammalogy, 94(4), pp. 792-800, 2013. DOI: 10.1644/12-MAMM-A-235.1

G. P. Rush, L. E. Clarke, M. Stone, M. J. Wood, “Can drones count gulls? Minimal disturbance and semiautomated image processing with an unmanned aerial vehicle for colony-nesting seabirds” Ecology and Evolution, 2018;8:12322-12334.

D. Schofield, A. Nagrani, A. Zisserman, M. Hayashi, T. Matsuzawa, D. Biro, and S. Carvalho, "Chimpanzee face recognition from videos in the wild using deep learning”, Sci. Adv. 2019;5: eaaw0736

G. Schofield, K. A. Katselidis, M. K. S. Lilley, R. D. Reina, and G. C. Hays, "Detecting elusive aspects of wildlife ecology using drones: New insights on the mating dynamics and operational sex ratios of sea turtles,” Functional Ecology, vol. 31, no. 12, pp. 2310-2319, Jun. 2017, DOI:10.1111/1365-2435.12930

H. Schmaljohann, "Radar aeroecology - a missing piece of the puzzle for studying the migration ecology of animals", Ecography 43: 236-238, 2020, doi: 10.1111/ecog.04807

Y. Shan, D. Paull, and R. I. McKay, "Machine learning of poorly predictable ecological data," Ecological Modelling, vol. 195, no. 1-2 pp. 129-138, May 2006, DOI:10.1016/j.ecolmodel.2005.11.015

C. S. Smith, J. H. Epstein, A. C. Breed, R. K. Plowright, K. J. Olival, C. de Jong, P. Daszak, and H. E. Field, "Satellite Telemetry and Long-Range Bat Movements,” PLoS ONE, vol. 6, no. 2, Feb. 2011

R. Steenweg, M. Hebblewhite, R. Kays, J. Ahumada, J. T. Fisher, C. Burton, S. E. Townsend, C. Carbone, J. M. Rowcliffe, J. Whittington, J. Brodie, J. A. Royle, A. Switalski, A. P. Clevenger, N. Heim and L. N. Rich "Scaling-up camera traps: 

DOI:10.1002/fee.1448.

P. M. Stepanian, P. B. Chilson and J. F. Kelly, “An introduction to radar image processing in ecology,” Methods in Ecology and Evolution, 5, pp. 730-738. 2014, DOI: 10.1111/2041-210X.12214

V. St-Louis, A. M. Pidgeon, T. Kuemmerle, R. Sonnenschein, V. C. Radeloff, M. K. Clayton, B. A. Locke, D. Bash, and P. Hostert, "Modelling avian biodiversity using raw, unclassified satellite imagery," Phil. Trans. R. Soc. B, vol. 369, no. 1643, pp. 1-10, May 2014. DOI: 10.1098/rstb.2013.0197

J. P. Suraci, M. Clinchy, B. Mugerwa, M. Delsey, D. W. Macdonald, J. A. Smith, C. C. Wilmers and L. Y. Zanette "A new Automated Behavioural Response into camera trap studies,” Methods in Ecology and Evolution, vol. 8, no. 8, 2016, DOI:10.1111/2041-210X.12711

L. Šver, A. Bielen, J. Križan and G. Gužvica, "Camera traps on wildlife crossing structures as a tool in Gray Wolf (Canis lupus) management - five-years monitoring of Wolf abundance trends in Croatia,” PLOS ONE, 11(16), e0156748. Jun. 2016, DOI:10.1371/journal.pone.0156748

K. R. R. Swinnen, J. Reijniers, M. Breno and H. Leirs, "A novel method to reduce time investment when processing videos from camera trap studies,” PLOS ONE, vol. 9, no. 6, Jun. 2014. e98881

M. A. Tabak, M. S. Norouzzadeh, D. W. Wolfson, S. J. Sweeney, K. C. Vercauteren, N. P. Snow, J. M. Halseth, P. A. Di Salvo, J. S. Lewis, M. D. White, B. Teton, J. C. Beasley, P. E. Schlichting, R. K. Boughton, B. Wight, E. S. Newkirk, J. S. Ivan, E. A. Odell, R. K. Brook, P. M. Lukacs, A. K. Moeller, E. G. Mandeville, J. Clune, R. S. Miller, "Machine learning to classify animal species in camera trap images: Applications in ecology," Methods Ecol Evol., 10, pp. 585590, 2019, DOI: 10.1111/2041-210X.13120

J. Troscianko, and C. Rutz, "Activity profiles and hook-tool use of New Caledonian crows recorded by bird-borne video cameras” Biol. Lett. 11: 20150777. http://dx.doi.org/10.1098/rsbl.2015.0777

J. J. Valletta, C. Torney, M. Kings, A. Thornton and J. Madden, "Applications of machine learning in animal behaviour studies,” Animal Behaviour, 124, pp. 203-220, 2017, DOI:10.1016/j.anbehav.2016.12.005

R. Van der Wal, C. Zeng, D. Heptinstall, K. Ponnamperuma, C. Mellish, S. Benn, and A. Siddharthan, "Automated data analysis to rapidly derive and communicate ecological insights from satellite-tag data: A case study of reintroduced red kites,” Ambio 44(Suppl. 4), pp. 612-623, 2015. DOI:10.1007/s13280-015-0711-3.

E. Vas, A. Lescroel, O. Duriez, G. Boguszewski and D. Gremillet, "Approaching birds with drones: first experiments and ethical guidelines,” Biol. Lett. 11: 20140754, DOI:10.1098/rsbl.2014.0754

K. Wang, S. E. Franklin, X. Guo and M. Cattet, "Remote sensing of ecology, biodiversity and conservation: A review from the perspective of remote sensing specialists,” Sensors, 10, pp. 9647-9667, 2010. DOI:10.3390/s101109647

B. G. Weinstein, “A computer vision for animal ecology,” J Anim Ecol. pp. 1-13, 2017. DOI: 10.1111/1365-2656.12780.

S. A. Wich, D. Dellatore, M. Houghton, R. Ardi and L. P. Koh, (2015) "A preliminary assessment of using conservation drones for Sumatran orangutan (Pongo abelii) distribution and density," Journal of Unmanned Vehicle Systems. ISSN 2291-3467

M. J. Wilber, W. J. Scheirer, P. Leitner, B. Heflin, J. Zott, D. Reinke, D. K. Delaney and T. E. Boult, "Animal recognition in the Mojave Desert: Vision tools for field biologists,” 2013 IEEE Workshop on Applications of Computer Vision (WACV), Tampa, FL, 2013, pp. 206-213, DOI: 10.1109/WACV.2013.6475020.

Wildlife Insights: https://www.wildlifeinsights.org/team-network 
M. Willi, R. T. Pitman, A. W. Cardoso, C. Locke, A. Swanson, A. Boyer, M. Veldthuis, L. Fortson, "Identifying animal species in camera trap images using deep learning and citizen science” Methods Ecol Evol. 2019;10:80-91, DOI: 10.1111/2041-210X.13099

J. Wäldchen, P. Mäder, “Machine learning for image based species identification”, Methods Ecol Eval. 2018;9:2216-2225.

X. Yu, J. Wang, R. Kays, P. A. Jansen, T. Wang and T. Huang, "Automated identification of animal species in camera trap images,” EURASIP Journal on Image and Video Processing 2013, 2013:52 